\title{
A Relativistic Model of Fluids Motion
}

\author{
Jaak Heinloo \\ Marine Systems Institute, Tallinn University of Technology \\ Akadeemia tee 21, Tallinn 12618, Estonia \\ Tel: 372-620-4317 E-mail: heinloo@phys.sea.ee
}

\begin{abstract}
The paper suggests a relativistic model of fluids motion combining the conventional formulation of the relativistic fluid mechanics with the "Maxwell's formulation" of equations of the relativistic flow field, following from application of the 4D formalism in deducing Maxwell's equations with the 4-potential replaced by the 4-momentum flux. The model is formulated to demonstrate a possibility of an alternative interpretation of viscosity effects in non-relativistic and relativistic situations. The model complements also the similarities in mathematical formulation of fluid mechanics and electrodynamics addressed in different contexts.
\end{abstract}

Keywords: Relativistic fluid dynamics

\section{Introduction}

The present paper discusses a model of relativistic fluid combining the conventional formulation of the relativistic fluid mechanics (RFM) with the 4D formalism applied in derivation of Maxwell equations in electrodynamics (ED) where the 4-potential is replaced by the 4-momentum flux. The combined model allows for a diverging treatment of the viscosity effect in classical (non-relativistic) and relativistic situations - while in the non-relativistic case the motion energy is accompanied with the energy dissipation to heat then in the relativistic case the role of heat appears played by an oscillating process described in appropriate terms by equations expressed similar to Maxwell equations in ED. The discussed model complements as well the commented in different contexts (Heinloo, 2009a; Marmanis, 1998; Sedov, 1987) analogy between mathematical formulations of fluid mechanics and electrodynamics (ED). It shows that the usage of the relativistic fluid mechanics instead of the non-relativistic fluid mechanics enhance the similarities.

\section{The model components}

\subsection{Conventional formulation of RFM}

Consider a flat space-time $x^{\alpha}=\left(x_{1}, x_{2}, x_{3}, c t\right)=(\boldsymbol{x}, c t)$ with the metrics $g_{11}=g^{11}=g_{22}=g^{22}=g_{33}=g^{33}=-1$, $g_{44}=g^{44}=1$ and $g_{\alpha \beta}=g^{\alpha \beta}=0$ when $\alpha \neq \beta \quad\left(c\right.$ is the light speed) and let $U^{\alpha}$,

$$
U^{\alpha}=\frac{d x^{\alpha}}{d \tau}=\left(\frac{v}{\sqrt{1-v^{2} / c^{2}}}, \frac{c}{\sqrt{1-v^{2} / c^{2}}}\right),
$$

denote the fluid 4-velocity in this space-time. In Eq. (1) $d \tau=d t \sqrt{1-v^{2} / c^{2}}$, where $v=|\boldsymbol{v}|$ and $\boldsymbol{v}=d \boldsymbol{x} / d t$, $\left(U^{\alpha} U_{\alpha}=c^{2}\right.$ ). Defining the energy-momentum tensor for the perfect fluids $T^{\alpha \beta}$ as $T^{\alpha \beta}=\rho^{0} U^{\alpha} U^{\beta}-p g^{\alpha \beta}$, in which $p$ is the pressure and $\rho^{0}$ is the rest mass density specified as $\rho^{0}=\mu^{0}+p / c^{2}$ where $\mu^{0}$ is the constant rest mass density for $p=0$, the motion equation for a non-perfect relativistic fluid would be formulated as (Anile, 1989; Moller, 1972; Landau \& Lifshitz, 1994)

$$
T_{, \beta}^{\alpha \beta}=f^{\alpha} .
$$

In Eq. (2) $f^{\alpha}=\left(\boldsymbol{f}, f^{4}\right)$ is the density of the 4-force acting on the medium due to the dissipative processes. The formulation of the relativistic FM in the form Eq. (2) requires the 4-force $f^{\alpha}$ to be specified. 


\section{2 "Maxwell's formulation" of the relativistic fluids flow field}

Consider tensor $F_{\alpha \beta}=\left(\rho^{0} U_{\alpha}\right)_{, \beta}-\left(\rho^{0} U_{\beta}\right)_{\alpha}$. Without any restriction we can write for $F^{\alpha \beta}$

$$
\left\{\begin{array}{c}
F_{\alpha \beta, \gamma}+F_{\beta \gamma, \alpha}+F_{\gamma \alpha, \beta}=0 \\
F^{\alpha \beta}{ }_{, \beta}=j^{\alpha}
\end{array}\right.
$$

where the first equation is the identity and the second equation defines the new quantity $j^{\alpha}$ instead of $\rho^{0} U_{\alpha}$. Defining the 3-vectors

$$
\boldsymbol{\varepsilon}=\left(\varepsilon_{1}=F_{41}, \varepsilon_{2}=F_{42}, \varepsilon_{3}=F_{43}\right)
$$

and

$$
\boldsymbol{Z}=\left(\boldsymbol{Z}^{1}=F_{32}, \boldsymbol{Z}^{2}=F_{13}, \boldsymbol{Z}^{3}=F_{21}\right)
$$

Eqs. (3) rewrites as

$$
\begin{aligned}
& \frac{1}{c} \frac{\partial \boldsymbol{\varepsilon}}{\partial}=\nabla \times \boldsymbol{z}-\boldsymbol{j} \\
& \frac{1}{c} \frac{\partial \boldsymbol{\varepsilon}}{\partial}=-\nabla \times \boldsymbol{\varepsilon} \\
& \nabla \cdot \boldsymbol{z}=0 \\
& \nabla \cdot \boldsymbol{\varepsilon}=c q,
\end{aligned}
$$

where $\boldsymbol{j}=\left(j^{1}, j^{2}, j^{3}\right)$ and $q=j^{4} / c$, coinciding in its written form with the Maxwell equations of ED (Anile, 1989; Landau \& Lifshitz, 1994) but differing from the latter by its physical sense. So, in our case $\mathcal{E}$ and $\boldsymbol{Z}$ are the flow field characteristics specified as

$$
\boldsymbol{\varepsilon}=\frac{1}{c}\left(\frac{\partial}{\partial t} \frac{\rho^{0} \boldsymbol{v}}{\sqrt{1-v^{2} / c^{2}}}+\nabla \frac{\rho^{0} c^{2}}{\sqrt{1-v^{2} / c^{2}}}\right)
$$

and

$$
\boldsymbol{B}=-\nabla \times \frac{\rho^{0} \boldsymbol{v}}{\sqrt{1-v^{2} / c^{2}}} .
$$

We shall call Eqs. (4) the "Maxwell's formulation" of the relativistic fluid flow field. Dissimilar to the conventional formulation of RFM, requiring specification of the 4-force acting on medium, the "Maxwell's formulation" requires specification of $j^{4}=(\boldsymbol{j}, c q)$.

\section{The model - combined formulation of RFM}

Consider the situation with the fluid moving particles rest mass conserved. In this case it follows from the condition $\left(\rho^{0} U^{\alpha}\right)_{, \alpha}=0$ that $U_{, \alpha}^{\alpha}=0$ and $T^{\alpha \beta}{ }_{, \beta}$ in Eq. (2) is expressed as

$$
T_{, \beta}^{\alpha \beta}=F^{\alpha \beta} U_{\beta} .
$$

On the other hand constituting, analogously to the electric current density in ED, that

$$
j^{\alpha}=\xi F^{\alpha \beta} U_{\beta}=\xi(c \boldsymbol{\varepsilon}+\boldsymbol{v} \times \boldsymbol{\mathcal { Z }}, \boldsymbol{\varepsilon} \cdot \boldsymbol{v}),
$$

with $\xi$ having the sense similar to the electric conductivity, we shall have

$$
f^{\alpha}=\xi^{-1} j^{\alpha} .
$$


Eq. (5) combines the conventional formulation of RFM with the "Maxwell's formulation" of relativistic fluids flow field given by Eqs. (4). In the combined description the "Maxwell's formulation" is attributed a role analogous to the thermodynamics in the non-relativistic case with one substantial difference - while in the non-relativistic case the internal energy is understood as irregular motion (heat) coupling the motion description with thermodynamics, then in the relativistic case the medium internal energy is understood as an oscillating process covered by Eqs. (4).

\section{Discussion}

The discussed model solves the following tasks. First, it points to a possibility of an alternative interpretation of viscosity effects in non-relativistic and relativistic situations and, secondly, it shows that the commented in different contexts similarities between the mathematical formulation of fluid mechanics and electrodynamics (Heinloo, 2009a; Marmanis, 1998; Sedov, 1987) enhance if instead of the non-relativistic fluid mechanics the relativistic fluid mechanics is utilized.

The solution of the first task removes the boundary condition problem to the equations of RFM accompanied with the formulation of the viscous term in these equations as a simple generalization of the viscous terms in the respective non-relativistic equations. The mentioned boundary condition problem explains why the applications of the RFM are restricted mostly to the perfect fluids, e.g. in the discussion of formation of shock waves in relativistic perfect fluid flows (Taub, 1978), where the problem of boundary conditions does not rise. We hope that the suggested model can be constructive for discussion, in particular, of cosmological problems e.g. related to the large-scale structure of the Universe as a stellar system, to the astrophysical plasma, etc. (Battaner, 1996) where the removal of the boundary condition problem may enhance the applicability of methods of relativistic fluid mechanics.

The second task enhances the analogy between the mathematical formulations of ED and of the fluid mechanics to the relativistic case. The systemic description of fluid mechanics (Heinloo, 2009) explains the discussed kind of analogy by the similarities in the compared fields' type, accompanied with their similar location in the conjoint systemic description. The discussed model insinuates that the similarities in mathematical formulations of the turbulence problem and of the description of fields of strong interaction (Heinloo, 2009a) also increase if the relativistic theory of turbulence (if formulated) was applied. Though the pointed kind of similarities does not spread to the described physical situations themselves, these similarities may suggest productive ideas. The definition of the turbulence spin, resulting in a substantial modernization of the average turbulence description (Heinloo, 2009a,b; 2008a,b; 2004), motivated by the analogy in mathematical formulations of turbulence problem and problems of fields of strong interaction, presents an example.

The author thanks Dr. Aleksander Toompuu for participation in discussions of the touched by the discussed model problems.

\section{References}

Anile, A. M. (1989). Relativistic fluids and magneto-fluids. Cambridge: Cambridge Univ. Press.

Battaner, E. (1996). Astrophysical Fluid Dynamics. Cambridge: Cambridge Univ. Press.

Heinloo, J. (2004). The formulation of turbulence mechanics. Phys. Rev E, 69, paper 056317.

Heinloo, J. (2008a). Setup of turbulence mechanics accounted for a preferred orientation of eddy rotation. Concepts of Phys., 5 (2), 205-219.

Heinloo, J. (2008b). The description of externally influenced turbulence accounting for a preferred orientation of eddy rotation. Eur. Phys. J. B, 62, 471-476.

Heinloo, J. (2009a). A setup of systemic description of fluids motion. Proc Estonian Acad. Sci., 58, 184-189.

Heinloo, J. (2009b). The structure of average turbulent flow field, Cent. Eur. J. Phys., 8 (1), 17-24.

Landau, L. D., \& Lifshitz, E. (1994). The Classical Theory of Fields (Course of Theoretical Physics, vol. 2). (4th ed.). Oxford: Pergamon Press.

Marmanis, H. (1998). Analogy between the Navier-Stokes equations and Maxwell's equations. Applications to turbulence. Physics of Fluids, 10, 1428-1437.

Moller, C. (1972). The Theory of Relativity. Oxford: Clarendon Press.

Sedov, L. I. (1971). A Course in Continuum Mechanics. Groningen: Wolters-Noordhoff.

Taub, A. H. (1978). Relativistic fluid mechanics. Ann. Rev. Fluid Mech., 10, 301-332. 